\title{
RACSAM
}

Rev. R. Acad. Cien. Serie A. Mat.

VOL. 102 (1), 2008, pp. 161,179

Geometría y Topología / Geometry and Topology

Artículo panorámico / Survey

\section{Equilibria in a class of games and topological results implying their existence}

\author{
Robert S. Simon, Stanisław Spież and Henryk Toruńczyk
}

\begin{abstract}
We survey results related to the problem of the existence of equilibria in some classes of infinitely repeated two-person games of incomplete information on one side, first considered by Aumann, Maschler and Stearns. We generalize this setting to a broader one of principal-agent problems. We also discuss topological results needed, presenting them dually (using cohomology in place of homology) and more systematically than in our earlier papers.

\section{Equilibrios en una clase de juegos y resultados topológicos que implican su existencia}

Resumen. Exponemos resultados relacionados con el problema de la existencia de equilibrios en algunas clases de juegos bipersonales infinitamente repetidos con información incompleta por una de las partes, considerados por primera vez por Aumann, Maschler y Stearns. Generalizamos este marco a uno más amplio de problemas de agentes principales. También discutimos los resultados topológicos necesarios, presentándolos dualmente (usando cohomología en lugar de homología) y de modo más sistemático que en nuestros artículos anteriores.
\end{abstract}

\section{Introduction}

In answer to a question of R. Aumann, M. Maschler and R. Stearns, the existence of equilibria in various classes of infinitely repeated games has been established by S. Sorin [19], J. Renault [14] and the present authors [17 [18]. The purpose of this paper is to survey results on infinitely repeated games related to the above-mentioned question and to outline the topological methods used. Also the existence of an equilibrium for certain principal-agent problems is demonstrated.

The paper is organized as follows. The survey on game theory is contained in Section 3 We show how the existence of equilibrium was proven for repeated games of incomplete information on one side using our topological results. An effort is being made to present the results surveyed so as to relate them to the issue of cooperation (in our case between 2 persons), and a new application is given in Section 4 There, we consider principal-agent situations where Nature chooses a state, informs the agent of this choice, the agent can send a signal to the principal and the two players can make contracts concerning the signals and joint actions. Under reasonable conditions concerning what contracts are acceptable to both parties there will be an acceptable contract in equilibrium.

Presentado por Pedro Jiménez Guerra.

Recibido: 30 de agosto de 2007. Aceptado: 5 de diciembre de 2007.

Palabras clave / Keywords: repeated games of incomplete information, principal-agent theory, sender-receiver games, incentive compatibility (Game Theory), essential mappings, coincidences, set-valued functions, cohomology (Topology)

Mathematics Subject Classifications: Primary 55M20, 91A20; Secondary 54C60, 54H25, 52A20, 91A05, 91A10, $91 \mathrm{~B} 52$.

(C) 2008 Real Academia de Ciencias, España. 
The topological results are discussed in Section 2 The basic notion is that of "property $\mathcal{S}$ " of a compact set $F \subset \mathbb{R}^{n} \times Y$. It is this property which allowed us to conclude that the projection of $F$ to $\mathbb{R}^{n}$ contained a given simplex — which for $F$ described by some complex conditions given by the game implied the existence of its equilibrium, as discussed in Section 3 The property $\mathcal{S}$ had been defined in [18], [17] in homological terms, but here we make an attempt to describe its cohomological analogue, which hopefully may become more handy for applications because of the richer structure of cohomology theory. At the same time we isolate as Propositions certain "axioms" for property $\mathcal{S}$ which make it useful for game-theoretic applications. These axioms have to do with cohomology (or homology) theory only inasmuch as they use the notion of acyclicity, that is of being equivalent to a point in this theory.

\section{The spanning property of correspondences}

\subsection{Cohomological properties of subsets of an $\mathbb{R}^{n}$}

Throughout the paper we denote by $\bar{C}$ or by $\operatorname{cl} C$ the closure of a set $C$, by int $C$ the interior of $C$ and by $\delta C$ the boundary of the interior of $C$. All topological spaces are assumed to be metrizable. We'll use the reduced Čech cohomology and homology functors with coefficients in an abelian group, Cf. [7] §IX.7]. If $h$ is one of these functors then $h\left(X, X_{0}\right)$ is considered as a graded group, i.e., as a direct sum of the $h^{n}\left(X, X_{0}\right)$ 's, and a specific group $h^{n}\left(X, X_{0}\right)$ is isolated only if needed; similarly for a homomorphism $h(f)$ induced by a map $f:\left(X, X_{0}\right) \rightarrow\left(Y, Y_{0}\right)$. As usual, $h(X)$ and $h(X, \emptyset)$ are identified. In subsections 2.12 .2 and 2.3 let $h$ stay for reduced cohomology with coefficients in a fixed abelian group.

In this subsection we discuss elementary properties of subsets of the space $\mathbb{R}^{n}$ on which the further results rest. We consider $\mathbb{R}^{n}$ as a subset of its one-point compactification $S^{n}$.

Lemma 1 Let $U$ be a connected, non-empty open set in $S^{n}$. Then:

(a). $h^{n}(\bar{U}, \delta U) \neq\{0\}$.

(b). $h^{n}(D, D \cap \delta U)=\{0\}$ for any proper closed subset $D$ of $\bar{U}$.

Proof. Ad (a) Excision of $S^{n} \backslash \bar{U}$ shows that $h^{n}(\bar{U}, \delta U)=h^{n}\left(S^{n}, S^{n} \backslash U\right)$. Hence by duality theorem we get $h^{n}(\bar{U}, \delta U)=H_{0}(U)$, the 0 -th singular homology group of $U$-which is non-trivial because $U \neq \emptyset$. (See [20, Theorem 17, page 296])

Ad(b) The same argument shows that if $D \supset \delta U$ then $h^{n}(D, \delta U)=H_{0}(U, U \backslash D)$-which is $\{0\}$, because $U$ is connected and $U \backslash D \neq \emptyset$. In the general case it remains to note that $h^{n}((D, D \cap \delta U) \hookrightarrow$ $(D \cup \delta U, \delta U))$ is an isomorphism, by excision, so we may replace $D$ by $D \cup \delta U$.

Lemma 2 Let $\left(D, D^{\prime}\right)$ and $\left(C, C^{\prime}\right)$ be compact pairs in $\mathbb{R}^{n}$ with $\left(C, C^{\prime}\right) \subset\left(D, D^{\prime}\right)$. If $D \backslash D^{\prime}$ is connected and open in $\mathbb{R}^{n}$ and is contained in a component of $C \backslash C^{\prime}$ which is open in $\mathbb{R}^{n}$, then the inclusion-induced homomorphism $h^{n}\left(\left(C, C^{\prime}\right) \hookrightarrow\left(D, D^{\prime}\right)\right)$ is injective.

Proof. Let $\alpha$ and $V$ be the homomorphism and the component of $C \backslash C^{\prime}$ in question. The inclusioninduced homomorphism $h^{n}\left(S^{n}, S^{n} \backslash\left(D \backslash D^{\prime}\right)\right) \rightarrow h^{n}\left(S^{n}, S^{n} \backslash V\right)$ is an isomorphism because it corresponds to the homomorphism $H_{0}\left(D \backslash D^{\prime} \hookrightarrow V\right)$ of singular homology groups. Therefore if $V=C \backslash C^{\prime}$ then excision of $V$ shows that $\alpha$ is an isomorphism.

In the general case we note that the above additional assumption is met when $C$ gets replaced by $C^{\prime} \cup V$, whence the inclusion-induced homomorphism $\gamma: h^{n}\left(D, D^{\prime}\right) \rightarrow h^{n}\left(C^{\prime} \cup V, C^{\prime}\right)$ is an isomorphism. The assertion follows, since $\gamma=\beta \circ \alpha$ for $\beta=h^{n}\left(\left(C^{\prime} \cup V, C^{\prime}\right) \hookrightarrow\left(C, C^{\prime}\right)\right)$.

Our last lemma here is a cohomological version of Borsuk's separation criterion [7] Theorem 3.6 on p. 302]: 
Lemma 3 Let $F$ be a compact subset of $\mathbb{R}^{n}$ not containing the origin. Then, $F$ separates 0 from $\infty$ if and only if the radial projection $p$ of $F$ to the unit sphere $S^{n-1} \subset \mathbb{R}^{n}$, defined by $p(x)=x /\|x\|$, has the property that $h(p): h\left(S^{n-1}\right) \rightarrow h(F)$ is a monomorphism.

PROOF. The separation property above means that $\mathbb{R}^{n} \backslash F$ is a disjoint union of two open sets, one of which is bounded and contains 0 . When $F$ fails to separate then it is well known that $p$ is null-homotopic and so $h(p)=0$. (See [7].)

Let us now assume $F$ separates this way and let $W$ denote the above bounded set. Let $D$ be a closed ball and $V$ an open ball, both centered at 0 , such that $F \subset D, V \subset W$ and $S^{n-1} \subset D \backslash V$. Let us consider the diagram

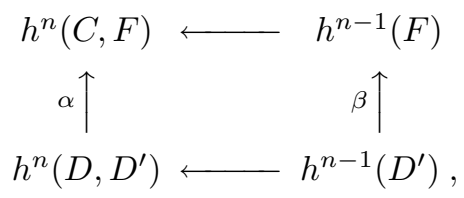

where $D^{\prime}:=D \backslash V, C:=F \cup W$, the vertical homomorphisms are induced by inclusions and the horizontal ones are coboundary homomorphisms. By Lemma $2 \alpha$ is injective. Moreover, $h^{n-1}\left(D^{\prime}\right) \rightarrow h^{n}\left(D, D^{\prime}\right)$ is an isomorphism and so $\beta: h^{n-1}\left(D^{\prime}\right) \rightarrow h^{n-1}(F)$ must be a monomorphism by the commutativity of the diagram. Consequently, $h^{n-1}(p)$ is a monomorphism as well, for $\beta=h^{n-1}(p) \circ \gamma$ with $\gamma=h^{n-1}\left(S^{n-1} \hookrightarrow\right.$ $\left.D^{\prime}\right)$ an isomorphism.

\subsection{Cohomological essentiality of mappings over subsets of $\mathbb{R}^{n}$}

Let us say that a map $f: X \rightarrow \mathbb{R}^{n}$ of a compact space $X$ is $h$-essential over a bounded set $T \subset \mathbb{R}^{n}$ if, with $U=\operatorname{int} T$, the $f$-induced homomorphism $h^{n}\left(\left(f^{-1}(\bar{U}), f^{-1}(\delta U)\right) \rightarrow(\bar{U}, \delta U)\right)$ is injective. (By a map we mean in this paper a continuous function.)

Remark 1 The above notion is equivalent to that of "property $\mathcal{S}$ " (of the correspondence $f^{-1}$ ) to be described in Section 2.3 but is more intuitive. We define it in analogy with the "essential maps onto cubes" of P. Alexandroff [1].

\section{Remark 2}

(a). Thus $f$ is $h$-essential over $T$ if and only if it is such over $U=\operatorname{int} T$.

(b). Also, $f$ is h-essential over $T$ if and only if $h^{n}\left(\left(f^{-1}(\bar{U}), f^{-1}(\delta T)\right) \rightarrow(T \cup \delta T, \delta T)\right)$ is injective. (This is so because $h^{n}((\bar{U}, \delta U) \hookrightarrow(T \cup \delta T, \delta T))$ is an isomorphism, as $\operatorname{dim}((T \cup \delta T) \backslash U)<n$.) We recall that $\delta T=\delta U$, the boundary of $U$.

(c). The cases when $T$ is compact or open are central to us. Clearly, $T \cup \delta T=\bar{T}$ when $T$ is open, and $T \cup \delta T=T$ when $T$ is compact.

Lemma 4 Let $X$ be a compact space, $f: X \rightarrow \mathbb{R}^{n}$ be a map and $U \subset \mathbb{R}^{n}$ be open and bounded.

(a). If $f$ is $h$-essential over each component of $U$ then it is essential over $U$.

(b). If $f$ is $h$-essential over $U$ then so it is over any open set $V \subset U$.

(c). If $f$ is h-essential over $U$ then $f(X) \supset \bar{U}$.

Proof. Let us write $X_{S}$ for $f^{-1}(S)$ when $S \subset \mathbb{R}^{n}$.

Ad (a) Suppose that $f$ is essential over each component of $U$ and let $\mathcal{V}$ be the family of all components of $U$. As noted earlier $h^{n}(\bar{U}, \delta U)$ is isomorphic to the singular homology group $H_{0}(U)$, which is isomorphic to $\prod\left\{H_{0}(V) \mid V \in \mathcal{V}\right\}$ and hence to $\prod\left\{h^{n}(\bar{V}, \delta V) \mid V \in \mathcal{V}\right\}$. It follows that the homomorphism 
$h^{n}(\bar{U}, \delta U) \rightarrow \prod\left\{h^{n}(\bar{V}, \delta V) \mid V \in \mathcal{V}\right\}$, given by the family $\left\{h^{n}((\bar{V}, \delta V) \hookrightarrow(\bar{U}, \delta U)) \mid V \in \mathcal{V}\right\}$, is an isomorphism. Now, let us consider the diagram

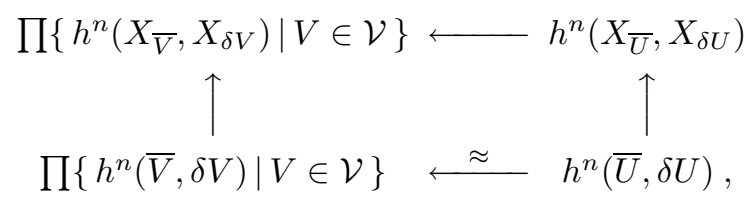

where the vertical homomorphisms are induced by $f$ and horizontal ones are given by families of inclusioninduced morphisms. Since the left vertical homomorphism is a monomorphism by assumption, so is the right one as well.

Ad(b) By part(a) we may assume additionally that the set $V$ is connected. (If it isn't, we treat each of its components individually.) We consider the diagram

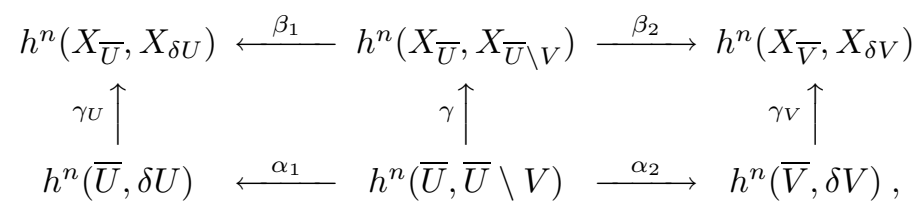

where horizontal homomorphisms are induced by inclusions and the vertical ones by $f$. By Lemma $\alpha_{1}$ is a monomorphism. Since $\gamma_{U}$ is a monomorphism either (by assumption), so is $\gamma$ by the commutativity of the left square of the diagram.

Moreover $\alpha_{2}$ and $\beta_{2}$ are isomorphisms by excision whence by using now the right square of the diagram it follows that $\gamma_{V}$ is a monomorphism, as desired.

Ad (c) Let $D=f\left(X_{\bar{U}}\right)$. The homomorphism witnessing the $h$-essentiality of $f$ over $U$ factors through the group $h^{n}(D, D \cap \delta U)$ and is defined on $h^{n}(\bar{U}, \delta U)$. Hence it follows from Lemma! that it could not be injective unless $D=\bar{U}$.

Lemma 5 Let a set $T \subset \mathbb{R}^{n}$ be bounded. For a map $f: X \rightarrow \mathbb{R}^{n}$ of a compact space $X$ the following conditions on a compact set $Z \subset f^{-1}(\delta T)$ are equivalent (below, the undefined homomorphisms are induced by $f$ ):

(a). $\operatorname{ker} h^{n}\left((X, Z) \rightarrow\left(\mathbb{R}^{n}, \delta T\right)\right) \subset \operatorname{ker} h^{n}\left((T \cup \delta T, \delta T) \hookrightarrow\left(\mathbb{R}^{n}, \delta T\right)\right)$;

(b). $\operatorname{ker}\left(\partial_{X} \circ h^{n-1}(Z \rightarrow \delta T)\right) \subset \operatorname{ker} \partial_{T}$, where $\partial_{X}$ is the coboundary homomorphism of the pair $(X, Z)$, and $\partial_{T}$ that of the pair $(T \cup \delta T, \delta T)$.

Moreover, $f$ is essential over $T$ if and only if there exists a compact set $Z \subset f^{-1}(\delta T)$ satisfying these conditions, in which case $f^{-1}(\delta T)$ satisfies them also.

PROOF. Observe that the coboundary $\partial: h^{n-1}(\delta T) \rightarrow h^{n}\left(\mathbb{R}^{n}, \delta T\right)$ is an isomorphism. Thus, the equalities $\partial_{X} \circ h^{n-1}(Z \rightarrow \delta T)=h^{n}\left((X, Z) \rightarrow\left(\mathbb{R}^{n}, \delta T\right)\right) \circ \partial$ and $\partial_{T}=h^{n}\left((T \cup \delta T, \delta T) \hookrightarrow\left(\mathbb{R}^{n}, \delta T\right)\right) \circ \partial$ imply the equivalence of (a) and (b)

Suppose now that $f$ is $h$-essential over $T$. The monomorphism from Remark (b) when right composed with $h^{n}\left((T \cup \delta T, \delta T) \hookrightarrow\left(\mathbb{R}^{n}, \delta T\right)\right)$, factors through $h^{n}\left(\left(X, f^{-1}(\delta T)\right) \rightarrow\left(\mathbb{R}^{n}, \delta T\right)\right)$. This yields (a) with $Z=f^{-1}(\delta T)$.

Finally, suppose that (a) holds for a compact set $Z \subset f^{-1}(\delta T)$; then it holds with $Z=f^{-1}(\delta T)$ as well. With $U=\operatorname{int} T$ we consider the following diagram

$$
\begin{array}{ccc}
h^{n}\left(X, f^{-1}(\delta U)\right) & \stackrel{\gamma^{\prime}}{\longrightarrow} & h^{n}\left(f^{-1}(\bar{U}), f^{-1}(\delta U)\right) \oplus h^{n}\left(f^{-1}\left(\mathbb{R}^{n} \backslash U\right), f^{-1}(\delta U)\right) \\
j \uparrow & & k=\left(k_{1}, k_{2}\right) \uparrow \\
\left.h^{n}\left(\mathbb{R}^{n}, \delta U\right)\right) & \stackrel{\gamma=\left(\gamma_{1}, \gamma_{2}\right)}{\longrightarrow} & h^{n}(\bar{U}, \delta U) \oplus h^{n}\left(\mathbb{R}^{n} \backslash U, \delta U\right),
\end{array}
$$


where $j$ and $k$ are induced by $f$, while $\gamma$ and $\gamma^{\prime}$ by inclusions (componentwise). Since $\bar{U} \cap\left(\mathbb{R}^{n} \backslash U\right)=\delta U$ and $f^{-1}(\bar{U}) \cap f^{-1}\left(\mathbb{R}^{n} \backslash U\right)=f^{-1}(\delta U)$ it follows from exactness of Mayer-Vietoris sequences for the couples of pairs $\left\{(\bar{U}, \delta U),\left(\mathbb{R}^{n} \backslash U, \delta U\right)\right\}$ and $\left\{\left(f^{-1}(\bar{U}), f^{-1}(\delta U)\right),\left(f^{-1}\left(\mathbb{R}^{n} \backslash U\right), f^{-1}(\delta U)\right)\right\}$, respectively, that $\gamma$ and $\gamma^{\prime}$ are isomorphisms. From this and the assumed inclusion $\operatorname{ker}(j) \subset \operatorname{ker}\left(\gamma_{1}\right)$ it easily follows that $\operatorname{ker}\left(k_{1}\right)=\{0\}$, i.e. $f$ is essential over $T$.

Definition 1 Let a set $T \subset \mathbb{R}^{n}$ be bounded. We say that $Z$ witnesses the h-essentiality over $T$ of a map $f: X \rightarrow \mathbb{R}^{n}$, if $Z$ is a compact subset of $f^{-1}(\delta T)$ and the equivalent conditions (a) (b) above are satisfied. Recall also that a mapping $f: X \rightarrow C$ is said to be $h$-acyclic if $h\left(f^{-1}(c)\right)=h(\{c\})$ for $c \in C$.

Lemma 6 Let $f: X \rightarrow \mathbb{R}^{n}$ be a map of a space $X=X_{1} \cup X_{2}$, where each $X_{i}$ is compact, and let $C_{1}$ and $C_{2}$ be compact sets in $\mathbb{R}^{n}$ such that int $C_{1} \cap \operatorname{int} C_{2}=\emptyset$. Then $f$ is h-essential over $C_{1} \cup C_{2}$ provided each $f \mid X_{i}$ is h-essential over $C_{i}$ and this is witnessed by compact sets $Z_{1}$ and $Z_{2}$, respectively, such that $f \mid Z_{1} \cap Z_{2}$ is an h-acyclic map onto $\delta C_{1} \cap \delta C_{2}$.

PROof. We consider the diagram below in which homomorphisms $j_{1}, j_{2}$ and $v$ are induced by $f$ and the remaining ones by suitable inclusions:

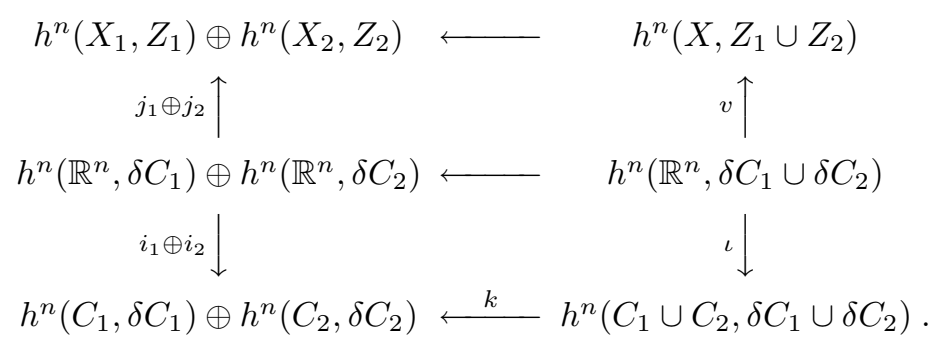

By assumption, $\operatorname{ker}\left(j_{s}\right) \subset \operatorname{ker}\left(i_{s}\right), s=1,2$, whence $\operatorname{ker}\left(j_{1} \oplus j_{2}\right) \subset \operatorname{ker}\left(i_{1} \oplus i_{2}\right)$. Moreover, since $\operatorname{dim}\left(C_{1} \cap C_{2}\right)<n$ the Mayer-Vietoris cohomology sequence of the couple of pairs $\left\{\left(C_{1}, \delta C_{1}\right),\left(C_{2}, \delta C_{2}\right)\right\}$ tells us that $k$ is a monomorphism. By commutativity of the diagram this implies that

$$
\operatorname{ker}(v) \subset \operatorname{ker}(\iota)
$$

We now let:

$$
\begin{aligned}
A:=\delta C_{1} \cup \delta C_{2}, \quad A_{1}:=\delta C_{1} \cap \delta C_{2}, \quad A_{2}=\delta\left(C_{1} \cup C_{2}\right), \quad A_{0}:=A_{1} \cap A_{2} \\
B:=Z_{1} \cup Z_{2}, \quad B_{1}:=Z_{1} \cap Z_{2}, \quad B_{2}:=f^{-1}\left(A_{2}\right) \cap B, \quad B_{0}:=B_{1} \cap B_{2} .
\end{aligned}
$$

Then $f\left(B_{k}\right) \subset A_{k}, A=A_{1} \cup A_{2}$ and $B=B_{1} \cup B_{2}$, so we get a commutative diagram

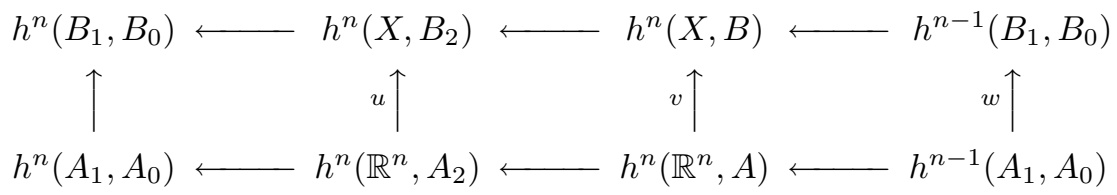

where vertical homomorphisms are induced by $f$ and rows are parts of the Mayer-Vietoris cohomology sequences of the couples of pairs $\left\{\left(X, B_{2}\right),\left(B_{1}, B_{1}\right)\right\}$ and $\left\{\left(\mathbb{R}^{n}, A_{2}\right),\left(A_{1}, A_{1}\right)\right\}$, respectively. (In these sequences we skip the trivial factors $h^{n}\left(B_{1}, B_{1}\right)$ and $h^{n}\left(A_{1}, A_{1}\right)$.)

We have $h^{n}\left(A_{1}, A_{0}\right)=0$, since $\operatorname{dim} A_{1}<n$. Thus, by exactness, the homomorphism $h^{n}\left(\mathbb{R}^{n}, A_{2}\right) \leftarrow$ $h^{n}\left(\mathbb{R}^{n}, A\right)$ is onto. By assumption, $f \mid B_{1}: B_{1} \rightarrow A_{1}$ is acyclic. Since additionally $B_{0}=f^{-1}\left(A_{0}\right) \cap B_{1}$ it 
follows from Vietoris mapping theorem that $w$ is an isomorphism. Hence, by $(*)$ and the commutativity of the inclusion-induced diagram

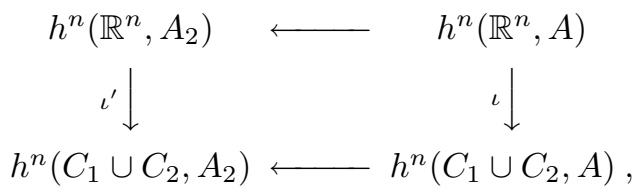

it follows from the Claim below that $\operatorname{ker}(u) \subset \operatorname{ker}\left(\iota^{\prime}\right)$. Thus the set $B_{2}$ witnesses $h$-essentiality of $f$ over $C_{1} \cup C_{2}$, what completes the proof.

Claim 1 Let the following diagram of homomorphisms of abelian groups

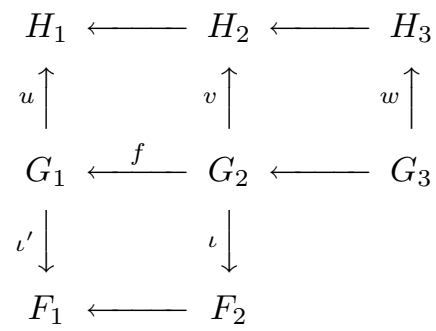

be commutative and have exact rows. If $f$ and $w$ are epimorphisms and $\operatorname{ker}(v) \subset \operatorname{ker}(\iota)$ then $\operatorname{ker}(u) \subset$ $\operatorname{ker}\left(\iota^{\prime}\right)$.

\subsection{Property $\mathcal{S}$ of (compact) correspondences: a cohomological version}

In this paper, by a correspondence $F: X \rightarrow Y$ we mean any compact subset of $X \times Y$. The image of $F$ in $Y \times X$ under the coordinate-switching map is denoted $F^{-1}$. If $X_{0}$ is a subset of $X$, then $F \cap\left(X_{0} \times Y\right)$ is called the restriction of $F$ to $X_{0}$ and denoted $F \mid X_{0}$. For a correspondence $F: X \rightarrow Y$, the image of the set $F \mid X_{0}$ under the projection to $Y$ is denoted by $F\left(X_{0}\right)$ and called the image of $X_{0}$ under $F$. We also write

$$
\operatorname{im}(F):=F(X), \quad F(x):=F(\{x\}) \text { for } x \in X \quad \text { and } \quad \operatorname{dom}(F):=\{x \in X: F(x) \neq \emptyset\} .
$$

Note that the set $\operatorname{dom}(F)$ is closed in $X$, as it is the image of a compact set $F$ under the projection $X \times Y \rightarrow X$. Clearly, a single-valued correspondence $X \rightarrow Y$ is a map (i.e., a continuous function).

If $F: X \rightarrow Y$ is a correspondence, it is not assumed a priori that $F(x) \neq \emptyset$ for all (or even for some) $x \in X$. In fact, the problem faced in game-theoretic applications is to establish, in the case when $X=\mathbb{R}^{n}$, whether $\operatorname{dom} F$ contains a given $n$-simplex $\Delta \subset \mathbb{R}^{n}$. The importance of such a conclusion is that it allows interpreting the non-emptiness of $F(p)$, for a suitably chosen correspondence $F$ and a point $p \in \Delta$, in terms of the existence of solutions to systems of inequalities encountered in game theory. The approach of handling this problem in [17] and [18] depended on the use of a certain homological property of correspondences $F: \mathbb{R}^{n} \rightarrow Y$ which implied that dom $F$ contained a given compact set. The aim of this subsection is to present a dual version of this property, defined cohomologically, and to isolate propositions which could be treated as "axioms" that make whether homological or cohomological versions useful in our applications.

Definition 2 Let $F: \mathbb{R}^{n} \rightarrow Y$ be a correspondence. We say that $F$ has property $\mathcal{S}$ for a set $T \subset \mathbb{R}^{n}$, and that a compact set $Z$ witnesses this, if $T$ is bounded and the projection $p: F \rightarrow \mathbb{R}^{n}$ has property $\mathcal{S}$ for $T$, it being witnessed by $Z$. 


\section{Remark 3}

(a). In view of the earlier definition, $F$ has property $\mathcal{S}$ for an open bounded set $U$ if and only if

$$
h^{n}(p:(F|\bar{U}, F| \delta U) \rightarrow(\bar{U}, \delta U)) \text { is a monomorphism. }
$$

(b). Above, "property $\mathcal{S}$ " stays for an abbreviation of the spanning property. This name is justified by part (a) of the following Proposition collecting some consequences of this property that follow easily either from definition and earlier results.

\section{Proposition 1}

(a). If $F$ has property $\mathcal{S}$ for an open set $U$, then $\operatorname{dom}(F) \supset \bar{U}$.

(b). If $C \supset D$ and $F$ has property $\mathcal{S}$ for $C$ then $F \mid D$ has it for $D$.

(c). If $F, G$ are correspondences such that $F \subset G$ and $F$ has property $\mathcal{S}$ for a set $C$, then $G$ has it either.

(d). F has property $\mathcal{S}$ for a compact set $C$ provided for every neighborhood $U$ of $\delta C$ in $\mathbb{R}^{n}$ and for every neighborhood $V$ of $F$ in $\mathbb{R}^{n} \times Y$ there exists a correspondence $F^{\prime}: \mathbb{R}^{n} \rightarrow Y$ such that $F^{\prime} \subset V$ and $F^{\prime}$ has property $\mathcal{S}$ for a compact set $C^{\prime}$ satisfying $\delta C^{\prime} \subset U$ and $C \backslash U \subset C^{\prime} \subset C \cup U$.

In particular, if each of the correspondences $F_{i}: \mathbb{R}^{n} \rightarrow Y$ has property $\mathcal{S}$ for $C$ and $F_{1} \supset F_{2} \supset F_{3} \ldots$ then $\bigcap_{n} F_{n}$ has this property also.

PRoof. Assertion [c) follows from the definition, while (a) and (b) follow from Lemma 4 (Part (b) reduces to the case when $C$ and $D$ are open.)

Ad (d) This follows from the continuity of the functor $h$.

Parts (c) and (d) imply:

Corollary 1 If a correspondence $F$ has property $\mathcal{S}$ for a compact set $C \subset \mathbb{R}^{n}$, then so does any compact correspondence $G \subset \mathbb{R}^{n} \times Y$ containing $F \mid \operatorname{int}(C)$.

When $Y=(0,1)$, property $\mathcal{S}$ for a simplex can easily be characterized:

Proposition 2 Let $\Delta$ be an n-simplex in $\mathbb{R}^{n}$. A correspondence $F: \Delta \rightarrow(0,1)$ has property $\mathcal{S}$ for $\Delta$ if and only if it separates $\Delta \times[0,1]$ between $\Delta \times\{0\}$ and $\Delta \times\{1\}$.

Proof. We assume that $\mathbb{R}^{n}$ is naturally embedded in its one point compactification $S^{n}$, which we consider to be the unit sphere of $\mathbb{R}^{n+1}$, and that $a, b \in(0,1)$ are such that $F \subset \Delta \times[a, b]$. The set $S^{n} \times[a, b]$ may be naturally identified with an annulus in $\mathbb{R}^{n+1}$ around 0 , and the set $\widetilde{F}:=F \cup\left(S^{n} \backslash\right.$ int $\left.\Delta\right) \times[a, b]$ —with a subset of this annulus. Then $F$ separates $\Delta \times[0,1]$ between $\Delta \times\{0\}$ and $\Delta \times\{1\}$ if and only if $\widetilde{F}$ separates $\mathbb{R}^{n+1}$ between 0 and $\infty$. The latter condition holds if and only if $h^{n}\left(p: \widetilde{F} \rightarrow S^{n}\right)$ is a monomorphism (by Lemma 3 which in turn by exactness and excision is equivalent to $h^{n}((\widetilde{F}|\Delta, \widetilde{F}| \delta \Delta) \rightarrow(\Delta, \delta \Delta))$ being a monomorphism. Since $F|\operatorname{int} \Delta=\widetilde{F}|$ int $\Delta$, the assertion follows from Corollary 1

Essential is the relation of property $\mathcal{S}$ to acyclicity. We employ the following notion: a correspondence $F: X \rightarrow Y$ is said to be acyclic-valued over a set $C \subset X$ if each set $F(x), x \in C$, is $h$-acyclic, i.e. satisfies $h(F(x))=h(\{$ point $\})$ (and hence is non-empty).

\section{Proposition 3}

(a). If a correspondence is acyclic-valued over a compact set $C$ then it has property $\mathcal{S}$ for $C$. 
(b). If correspondences $F, G: \mathbb{R}^{n} \rightarrow Y$ and compact sets $C, D \subset \mathbb{R}^{n}$ are such that $F$ has property $\mathcal{S}$ for $C$ and $G$ has it for $D$, then $F \cup G$ has it for $C \cup D$ provided additionally $G$ is acyclicvalued over $D \cap \delta C$ and the property $\mathcal{S}$ of $F$ for $C$ is being witnessed by a compact set $Z$ such that $Z \mid D \cap \delta C \subset G$ 回

Proof. Ad (a) This follows from Vietoris theorem (see [20 Theorem 15, p. 344]).

Ad (b) We apply Lemma 6 with $X_{1}=F \cup\left(G \mid \delta C_{1} \cap \delta C_{2}\right), X_{2}=G, C_{1}=C, C_{2}=D \backslash \operatorname{int} C$ and $f$ being the restriction of the projection along the $Y$-axis. The witnesses $Z_{i}$ are defined as follows: $Z_{1}=Z \cup\left(G \mid \delta C_{1} \cap \delta C_{2}\right)$ and $Z_{2}=G \mid \delta C_{2}$. Then, the assumptions of lemma 6 are satisfied and its assertion implies the desired property of $F \cup G$.

Proposition 4 Let $U \subset \mathbb{R}^{n}$ be open and bounded and $F, G: \mathbb{R}^{n} \rightarrow Y$ be correspondences satisfying $F \mid \delta U \subset G$. Suppose further that $F$ has property $\mathcal{S}$ for $U$ and $G^{-1}$ is acyclic-valued over $\operatorname{im}(G)$. If $\operatorname{dim} F(U)<n$ then $G$ has property $\mathcal{S}$ for $U$, it being witnessed by $F \mid \delta U$.

Proof. We may replace $G$ by a smaller correspondence $G \cap\left(\mathbb{R}^{n} \times F(\delta U)\right)$ and thus assume that $G \subset$ $\mathbb{R}^{n} \times F(\delta U)$. By lemma 5 applied to $F \mid \bar{U}$,

$$
\operatorname{ker}\left(\partial_{F} \circ h^{n-1}(F \mid \delta U \rightarrow \delta U)\right) \subset \operatorname{ker} \partial
$$

where $\partial: h^{n-1}(\delta U) \rightarrow h^{n}(\bar{U}, \delta U)$ and $\partial_{F}: h^{n-1}(F \mid \delta U) \rightarrow h^{n}(F|\bar{U}, F| \delta U)$ are coboundary homomorphisms. Suppose we knew that $\partial_{F} \circ \iota=0$, where $\iota=h^{n-1}(F \mid \delta U \hookrightarrow G)$. Then ker $\partial_{G}=\operatorname{im} \iota \subset \operatorname{ker} \partial_{F}$, where $\partial_{G}: h^{n-1}(F \mid \delta U) \rightarrow h^{n}(G, F \mid \delta U)$ is the coboundary homomorphism. With $(*)$ this would give $\operatorname{ker}\left(\partial_{G} \circ h^{n-1}(F \mid \delta U \rightarrow \delta U)\right) \subset \operatorname{ker} \partial$, which by Lemma 5 is equivalent to the assertion.

Thus it remains to show that $\partial_{F} \circ \iota$ is trivial, and to this end we consider the following commutative diagram

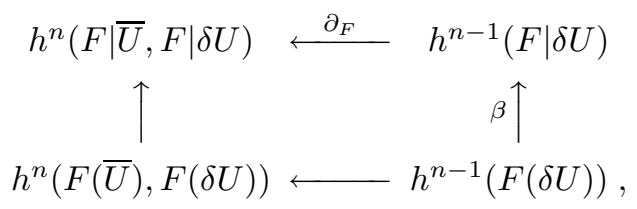

where horizontal homomorphisms are coboundary homomorphisms and the vertical ones are induced by projections along the $\mathbb{R}^{n}$-axis. Note that $h^{n}(F(\bar{U}), F(\delta U))=0$ since $\operatorname{dim}(F(U))<n$. It follows that $\partial_{F} \circ \beta=0$.

Now, $\beta$ is the composition of $\iota$ with the homomorphism $h^{n-1}(F(\delta U)) \rightarrow h^{n-1}(G)$ induced by projection along the $\mathbb{R}^{n}$-axis. The latter is an isomorphism by Vietoris theorem, and hence from $\partial_{F} \circ \beta=0$ it follows that $\partial_{F} \circ \iota=0$, as desired.

\subsection{Property $\mathcal{S}$ : a homological version}

The approach of the previous subsection may easily be dualized. That is, with $h$ now denoting the reduced Čech homology functor with coefficients in a compact abelian group, let us dualize the definition of property $\mathcal{S}$ (see below), while keeping intact the definitions of $h$-acyclicity of a compact set and of an acyclic-valued correspondence. (To be strict the latter should be called $h$-acyclic-valued, but the choice of $h$ is assumed to be clear or irrelevant.)

Definition 3 With h as above, a correspondence $F: \mathbb{R}^{n} \rightarrow Y$ is said to have property $\mathcal{S}$ for an open set $U \subset \mathbb{R}^{n}$ if $U$ is bounded and

$$
h_{n}(p:(F|\bar{U}, F| \delta U) \rightarrow(\bar{U}, \delta U)) \text { is an epimorphism. }
$$

\footnotetext{
${ }^{1}$ In part c) of [18 Lemma 2], which corresponds to a weaker version of this statement, there is a misprint: instead of "property $\mathcal{S}$ of $F$ for $U_{1}$ " there should be "property $\mathcal{S}$ of $F$ for $\bar{U}_{1}$ "
} 
Moreover if for a given compact set $Z \subset F \mid \delta U$

$$
\operatorname{im} h_{n}\left(p:(F, Z) \rightarrow\left(\mathbb{R}^{n}, \delta U\right)\right) \supset \operatorname{im} h_{n}\left((\bar{U}, \delta U) \hookrightarrow\left(\mathbb{R}^{n}, \delta U\right)\right)
$$

then we say that $Z$ witnesses the property $\mathcal{S}$ for $U$. (Above, $p$ is the projection along the $Y$-axis.) We also say $F$ has property $\mathcal{S}$ for a bounded set $T \subset \mathbb{R}^{n}$, witnessed by a compact set $Z \subset F \mid \delta T$, if this is true with $T$ replaced by int $T$.

Proposition 5 The previous propositions remain valid with the above definition of property $\mathcal{S}$, as does also Corollary 1

To see this, one may reverse all arrows and interchange words "surjective" and "injective" in the proofs of these propositions given in subsection 2.3 These proofs depend only on lemmas 1 to 6 the homological versions of which remain valid (with definition of homological essentiality being obtained from that of subsection 2.2 by a similar dualization). The proofs of lemmas 1 to 4 however, need to be given anew, for the duality theorem we used is not self dual. Another method of proving the Propositions is given in [18].

Remark 4 The approach in [18] was somewhat different. To recall it let us denote the $n-$ sphere $\mathbb{R}^{n} \cup\{\infty\}$ by $S^{n}$ and for an open bounded set $U$ let $[\bar{U}, \delta U]$ be the image of an orientation class $\omega \in h\left(S^{n}\right)$ under the composition

$$
h\left(S^{n}\right) \rightarrow h\left(S^{n}, S^{n} \backslash U\right) \rightarrow h(\bar{U}, \delta U),
$$

where the first homomorphism is induced by inclusion and the second one by excision of int $\left(S^{n} \backslash U\right)$. The property of a correspondence $F: \mathbb{R}^{n} \rightarrow Y$ isolated in $[18]$ demanded that $[\bar{U}, \delta U]$ be in the image of the projection-induced map $h_{n}((F|\bar{U}, F| \delta U) \rightarrow(\bar{U}, \delta U))$. This, however, turns out to be equivalent to the homological property $\mathcal{S}$ defined above. We skip the proof, which involves the fact that $[\bar{U}, \delta U]$ generates $h_{n}(\bar{U}, \delta U)$ when $U$ is connected.

To summarize, both cohomological and homological versions of property $\mathcal{S}$ can be defined. We'll see however that there is no real need to distinguish between them, however, as far as current applications go: these rely only on Propositions 14 (and on their consequences) which hold true for both versions.

\subsection{Applications to saturated correspondences}

In the sequel when $T$ and $L$ are given sets, with $L$ finite, then $T^{L}$ denotes the product $\prod_{l \in L} T_{l}$, where $T_{l}=T$ for each $l$. If $L$ is a subset of a set $K$ then for $x \in \mathbb{R}^{K}$ we denote by $x^{L}$ the natural projection of $x$ to $\mathbb{R}^{L}$; in particular, $x^{l}$ is the $l$-th coordinate of $x$. We equip the space $\mathbb{R}^{L}$ with the dot scalar product $x \cdot y=\sum_{l} x^{l} y^{l}$. By $\Delta$ or by $\Delta(L)$ we denote the simplex

$$
\Delta(L)=\left\{p=\left(p^{l}\right)_{l \in L} \in[0,1]^{L}: \sum_{l} p^{l}=1\right\}
$$

and we consider $\Delta(L)$ as a subset of $\Delta(K)$ by identifying each $x \in \Delta(L)$ with the (unique) point $\tilde{x} \in \Delta(K)$ such that $\tilde{x}^{L}=x$.

For applications to game theory, "saturated" correspondences into cubes turn out to be special:

Definition 4 If $F: \Delta(L) \rightarrow Y$ is a correspondence and $Y \subset \mathbb{R}^{L}$, then by $F^{+}$we denote the correspondence $\Delta(L) \rightarrow Y$ defined by

$$
F^{+}(p):=\left\{y \in Y: \exists x \in F(p) \text { such that } x^{l} \leq y^{l} \text { for all } l \in L \text { and } x^{l}=y^{l} \text { if } p^{l}>0\right\} .
$$

We call $F^{+}$the $Y$-saturation of $F$ and say that $F$ is saturated if $F=F^{+}$. Below, $Y=I^{L}$ is a cube, with I a non-trivial compact segment in $\mathbb{R}$. 
Theorem 1 Let $\mathcal{L}$ be a family of non-void subsets of a finite set $K$ such that $\bigcup \mathcal{L}=K$. Suppose further there are given a point $p \in \Delta(K)$ and, for every $L \in \mathcal{L}$, a saturated correspondence $F_{L}: \Delta(L) \rightarrow I^{L}$ with property $\mathcal{S}$ for $\Delta(L)$ and a closed convex subset $U_{L}$ of $I^{K}$ containing the point $(b, b, \ldots, b)$. Then there exist a point $y \in \bigcap_{L \in \mathcal{L}} U_{L} \subset I^{K}$ and finitely many sets $L_{1}, \ldots, L_{s} \in \mathcal{L}$ and points $p_{i} \in \Delta\left(L_{i}\right) \subset \Delta(K)$, $i=1, \ldots, s$, such that the following conditions hold:

$$
p \in \operatorname{conv}\left\{p_{1}, \ldots, p_{s}\right\} \text { and } y^{L_{i}} \in F_{L_{i}}\left(p_{i}\right) \text { for every } i=1, \ldots, s .
$$

To warrant property $\mathcal{S}$ of the correspondences $F_{L}$ we depend on the following

Theorem 2 Let $F: \Delta \rightarrow I^{L}$ be a convex-valued correspondence and $a: \Delta \rightarrow I$ be a lower semicontinuous function such that

$$
a(q) \leq \sup \{y \cdot q: y \in F(p)\} \quad \text { for all } p, q \in \Delta .
$$

With $F^{+}$denoting the $I^{L}$-saturation of $F$, the correspondence $\widetilde{F}: \Delta \rightarrow \mathbb{R}^{L}$ defined by the formula below has property $\mathcal{S}$ for $\Delta$ :

$$
\widetilde{F}(p):=\left\{y \in c\left(F^{+}\right)(p): y \cdot q \geq a(q) \text { for all } q \in \Delta\right\} .
$$

Above, convex-valued means that each set $F(p), \quad p \in \Delta$, is non-empty and convex, while $c\left(F^{+}\right)$ denotes the levelwise convexification of the correspondence $G=F^{+}$-by which we mean the set $\bigcup_{y \in I^{L}} \operatorname{conv}\left(G^{-1}(y)\right) \times\{y\} \subset \Delta \times I^{L}$. Let us note that $c\left(F^{+}\right)(p)$ consists of all points $y \in I^{L}$ such that for some $p_{1}, \ldots, p_{s} \in \Delta$ one has $p \in \operatorname{conv}\left\{p_{i}\right\}_{i=1}^{s}$ and $y \in F^{+}\left(p_{i}\right)$ for $i=1, \ldots, s$.

These two results are the consequences of Propositions from 1 to 4 needed in game theory. Their proofs, given in [18] (see Corollary 2 and Theorem 1 there), depend on the truth of Propositions from 1 to 4 but not on the definition of property $\mathcal{S}$. The proof of Theorem 1 leads also to the following characterization of certain saturated correspondences having property $\mathcal{S}$ :

Theorem 3 Let $F: \Delta(K) \rightarrow I^{K}$ be a saturated correspondence such that for each $y \in \operatorname{im}(F)$ the set $F^{-1}(y)$ is acyclic. Then, $F$ has property $\mathcal{S}$ for $\Delta(K)$ if and only if, with $J=[a, b]$ denoting a segment such that $I \subset(a, b)$, the image of the $J^{K}$-saturation of $F$ separates the cube $J^{K}$ between the vertices $v_{+}=(b, \ldots, b)$ and $v_{-}=(a, \ldots, a)$.

\subsection{Relation to antipodal-type theorems}

From Proposition 4 applied to a single-valued function it follows that when $x_{0}$ is a point of a compact set $C \subset \mathbb{R}^{n}$ and $f: C \rightarrow Y$ is a mapping into a space of dimension $n-1$, then in the boundary of $C$ there exists a set $C_{0}$ mapped by $f$ into a singleton and containing $x_{0}$ in its convex hull. By Caratheodory's theorem one can always replace $C_{0}$ by its subset consisting of $\leq n+1$ points. In general, this number cannot be lowered; an example is given in [9] and [17, p. 6]. However, in the special case where $Y$ is an $(n-1)$-manifold, a generalization of Borsuk-Ulam theorem given by Olędzki [13] implies that $C_{0}$ may be taken to consist of 2 points. (Special cases of this were established in [16] and [10]. The well-known Borsuk-Ulam theorem deals with the case when $C$ is a ball.)

It would be interesting to know less restrictive assumptions under which $n+1$ above could be replaced by a smaller number. This is related also to estimating the $k$-Urysohn diameter of a compact set $C$, defined as the infimum of $\sup \left\{\operatorname{diam}\left(f^{-1}(y): y \in f(C)\right\}\right.$ where $f$ runs over all mappings of $C$ to $k$-dimensional spaces. It follows easily from the above and the examples in [9 17], that the $(n-1)$-diameter of a ball in $\mathbb{R}^{n}$ equals to the "usual" diameter of a regular simplex inscribed into this ball's boundary (apparently this has already been known). When $k \in(n / 2, n-2]$ the $k$-Urysohn diameter of an $n$-ball remains unknown. For more information see [15, 21] and the references quoted there. 


\section{Repeated Games of Incomplete Information on One Side}

\subsection{Introduction}

One-shot games of incomplete information on one side were first introduced by J. Harsanyi [8] and the infinitely repeated ones of this type by R. Aumann and M. Maschler [2], both in the middle 1960's; further basic results relevant to this section were obtained in [5](1968) and [19](1983). The specific case of infinitely repeated two-person, non-zero-sum games of incomplete information on one side, described below, was introduced by R. Aumann, M. Maschler, and R. Stearns [5], which was a technical report to the U.S. Disarment Agency. The papers [2] 3] 5] got reprinted in [4], published in 1995.

There is a finite set $K$ of states of nature and two players. Nature chooses a state $k \in K$ according to a commonly known probability distribution on $K$. The first player, but not the second player, is informed of nature's choice. The finite sets of moves for the players are the same for all states. The chosen state remains constant throughout the play. Although the chosen state $k$, along with the moves of the players, determines the stage payoffs, during the play the second player learns nothing about his payoff, as this could give him information about the state of nature.

Let $m$ be the number of the first player's actions and $n$ the number of the second player's actions. For every state $k \in K$ there are two $m \times n$ matrices $A^{k}$ and $B^{k}$. The $i, j$ entry of $A^{k}$ is the payoff that the first player receives if the state of nature is $k$, the first player chooses the action $i$ and the second player chooses the actions $j$. Likewise the $i, j$ entry of $B^{k}$ is the payoff that the second player receives if the state of nature is $k$, the first player chooses the action $i$ and the second player chooses the actions $j$.

An equilibrium of the game is a pair of strategies such that for every state $k$ there are limits $a^{k}$ and $b^{k}$ as the number $n$ of stages go to infinity for the averages summed over the stages up to the stage $n$ of the expected payoffs of Players One and Two, respectively, and neither player can obtain a higher limit superior as $n$ goes to infinity for his average payoff summed over the stages up to $n$ (and determined by the initial probability distribution on $K$ ) by choosing a different strategy. One should not define the payoffs as the expected limit superior taken first on each state independently, because then erratic behavior by the second player could result in meaningless payoffs.

We divide the problem of equilibrium existence for these games into four levels of difficulty.

The first level of difficulty concerns the conventional game (of standard information): after each stage of play both players are informed of each others' moves and this is the only information the players receive additional to what they knew when the play began. Equilibrium existence for this level was proven in [17], c.f. $|19|$.

For the second level of difficulty both players do not know exactly what the other player has done, but at least the perception of the second player is independent of the state. Equilibrium existence for the second level was proven by J. Renault [14].

For the third level of difficulty the perception of the second player could be dependent on the state, however the first player has at least some channel with which she can communicate messages that reveal nothing about the state. With the first and second levels of difficulty the second player gains information on the state only from inferences obtained from the behavior of the first player. But with the third level of difficulty the second player's opportunity to learn about the state is more complex. Equilibrium existence for the third level was established in [18].

For the fourth level of difficulty there are no assumptions whatsoever concerning the perception of the second player. The question of equilibrium existence for the fourth level remains open.

The primary difficulty in establishing equilibrium existence for all levels concerns the ability of the first player to deceive. By an opportunity for deception we mean that Player One can act initially as if the state is something different from what it is, with initial unfavorable payoffs for her, in order to convince the second player to behave in the future in a way that is very favorable to her. A typical real-life example of such behavior would be that of "pool sharking". If we allow the game to be repeated only finitely many times (or allow for infinite repetition but introduce a discount factor) equilibrium existence is not problematic because 
the payoffs will be continuous functions on compact strategy spaces. With such equilibria the first player's payoff is a sum giving positive weight to all stages. Deception here involves a balancing act; the deceiver takes an initial loss to be balanced by a future gain. But when an infinitely repeated game is un-discounted there is no balancing act since the payoffs are always determined by the tail behavior, similar to a "last" game with stakes that far exceed those of the preceding games combined.

\subsection{An Example}

As an introduction to these games, let us consider an example that was instrumental in the discovery of the proof of equilibrium existence (for the first level of difficulty).

Example 1 There are three states of nature, labelled 1,2, and 3. Due to a high degree of symmetry in the definition of the game, the set $\{1,2,3\}$ is used to define the states and the moves of both players. Player One has three moves, 1, 2, and 3, and Player Two also has three moves 1, 2, and 3. All statements are respective to modulo 3 . Nature chooses the state of nature with a $1 / 3$ probability for all three states.

Player Two's payoff is very simple: no matter what Player One does Player Two receives a payoff of 1 if the state of nature matches his move, meaning that if the state of nature is $i$ and his move is also $i$ then Player Two gets a payoff of 1 , and otherwise Player Two receives a payoff of 0 .

The payoff for Player One is more complex. We describe it for any state of nature $i$.

Given that Player Two chooses the move $i$ if Player One chooses the move $i$ then she gets -4 if Player One chooses the move $i+1$ then she gets 0 if Player One chooses the move $i-1$ then she gets 0 .

Given that Player Two chooses the move $i+1$ if Player One chooses the move $i$ then she gets -4 if Player One chooses the move $i+1$ then she gets 1 if Player One chooses the move $i-1$ then she gets 2 .

Given that Player Two chooses the move $i-1$ if Player One chooses the move $i$ then she gets -4 , if Player One chooses the move $i+1$ then she gets 2, if Player One chooses the move $i-1$ then she gets 1 .

On the first level of analysis, Player Two wants his move to match the state, Player One wants to avoid matching her move with the state.

How shall we understand the dilemma facing Player One? Since one of her moves is poisonous to her, the $i$ th move if the state of nature is $i$, we expect that long term behavior of avoiding the move $i$ will suggest to Player Two that the state is $i$. The only way to reveal no information to Player Two concerning the state of nature would be to perform all moves with almost equal probability. If Player $i$ chose each move with $1 / 3$ probability then no matter what Player Two does Player One would receive an expected payoff of no more than $-1 / 3$.

On the other hand, let us assume that Player One always tells Player Two (through her choice of moves) what is the true state of nature. The natural way to do this, if the state of nature is $i$, would be for Player One to spend the rest of the time playing the moves $i+1$ and $i-1$, and Player Two will play $i$. The expected payoff for Player One would be 0 , no matter what. Therefore it makes no sense for Player One to act as if all states were equally likely. Have we now found an equilibrium?

The answer is no, because Player One has something much better to do than this. Now we describe an equilibrium. If the state of nature is $i$, then with one-half probability Player One will play only the move $i+1$ in the future and with one-half probability Player One will play only the move $i-1$ in the future. What will result from such a strategy? Let us assume that Player One chose the move $i$. By Bayesian 
analysis of conditional probability there is a $1 / 2$ probability that $i-1$ is the state of nature and there is a $1 / 2$ probability that $i+1$ is the state of nature. Now matter how Player Two responds the average payoff will be 1 for Player One. However Player Two could give her a payoff of 0 for one of the two states and a payoff of 2 for the other. Assuming that Player One chooses the move $i$, a reasonable response from Player Two would be to play the moves $i-1$ and $i+1$ with equal probability. The result would be a payoff of exactly 1 for Player One at both states and 1/2 for Player Two. Since Player One would do much worse if she played the move $i$ when the state was $i$, we have described an equilibrium. But notice that the behavior of Player Two is critical for the equilibrium property. If Player Two did not play $i+1$ and $i-1$ with equal probability then indeed Player One could have had a motivation to always choose one of the moves over the other.

\subsection{First Level of Difficulty}

We keep notation of subsection 2.5 For every $p \in \Delta(K)$ we define the matrix $A(p)=\sum_{k \in K} p^{k} A^{k}$, and the same for $B(p)$. For any function $f$ on a convex set let $\operatorname{cav}(f)$ (respectively $\operatorname{vex}(f)$ ) be the smallest concave function (largest convex function) larger or equal to (smaller or equal to) the function $f$. When multiplying a matrix on the right side by a vector, we assume that the vector is in vertical form. We define the function $a^{*}: \Delta(K) \rightarrow \mathbb{R}$ by

$$
a^{*}(q):=\max _{\sigma \in \Delta(I)} \min _{\tau \in \Delta(J)} \sigma A(q) \tau=\min _{\tau \in \Delta(J)} \max _{\sigma \in \Delta(I)} \sigma A(q) \tau
$$

and $b^{*}: \Delta(K) \rightarrow \mathbb{R}$ by

$$
b^{*}(p):=\min _{\sigma \in \Delta(I)} \max _{\tau \in \Delta(J)} \sigma B(p) \tau=\max _{\tau \in \Delta(J)} \min _{\sigma \in \Delta(I)} \sigma B(p) \tau .
$$

Assuming that the game is zero-sum, meaning that $B^{k}=-A^{k}$ for all $k \in K$, Aumann and Maschler determined in [4] that the value of the un-discounted infinitely repeated game is the function value of $\operatorname{cav}\left(a^{*}\right)$ applied to the initial probability. The most important part of this proof, based on [6], was to show that for any vector $x \in \mathbb{R}^{K}$ such that $x \cdot q \geq a^{*}(q)$ for all $q \in \Delta(K)$ the second player has a strategy such that with probability one for every state $k \in K$ and every strategy of the first player the limit superior of the first player's average payoff is no more than $x^{k}$. Such a vector $x \in \mathbb{R}^{K}$ we define to be individually rational for Player One. For any finite set $L$ and function $f: \Delta(L) \rightarrow \mathbb{R}$ a vector $x \in \mathbb{R}^{K}$ dominates $f$ if $x \cdot q \geq f(q)$ for all $q \in \Delta(L)$.

For every $\gamma \in \Delta(I \times J)$ define $\gamma A \in \mathbb{R}^{K}$ by $(\gamma A)^{k}:=\sum_{(i, j) \in I \times J} \gamma^{(i, j)} A^{k}(i, j)$ and define $\gamma B$ likewise.

A joint plan is a cooperative agreement between the players to perform a prescribed sequence of moves determined by a signal given by the informed player. Because the game is non-cooperative by definition, any cooperative agreement must be enforced by a threat of punishment. For an initial probability $p_{0}$ on the states of nature a joint plan is generated by

1. a finite subset of (posterior) probabilities $V \subseteq \Delta(K)$ such that the convex hull of $V$ contains the initial probability $p_{0}$,

2. for every $v \in V$ a $\gamma_{v} \in \Delta(I \times J)$,

3. for some finite $n$ a finite set $S \subset I^{n}$ of signals in bijective relation to the set $V$ and a choice by lottery, dependent on the state of nature and performed by Player One, of a member of $S$ in the first $n$ moves such that the signal $s \in S$ implies by Bayes rule a conditional probability on the set $K$ equal to the member of $V$ corresponding bijectively to $s$,

4. if the signal $s$ chosen corresponds to $v \in V$, an agreement between the players to play through the rest of the game a deterministic sequence of pairs of moves $\left(\left(i_{1}, j_{1}\right),\left(i_{2}, j_{2}\right), \ldots\right)$ such that in the limit the distribution $\gamma_{v}$ is obtained, and 
5. punishment strategies of the two players to be implemented in the event that a player does not adhere to the agreed upon sequence of moves. When the first player punishes the second player, the second player expects to receive no more than $\operatorname{vex}\left(b^{*}\right)(v)$, where $v \in V$ is the corresponding posteri or probability. When the second player punishes the first player, it is according to a individually rational vector $y \in \mathbb{R}^{K}$, as described above.

A joint plan is non-revealing if and only if $V=\left\{p_{0}\right\}$, where $p_{0}$ is the initial probability on the states of nature.

The joint plan describes an equilibrium of the un-discounted non-zero-sum game if ([4]) there is an individually rational $y \in \mathbb{R}^{K}$ such that for every $v \in V$ the following holds:

1. $\left(\gamma_{v} B\right) \cdot v \geq \operatorname{vex}\left(b^{*}\right)(v)$,

2. $\forall k \in K\left(\gamma_{v} A\right)^{k}=y^{k}$ if $v^{k}>0$,

3. $\forall k \in K\left(\gamma_{v} A\right)^{k} \leq y^{k}$ if $v^{k}=0$.

The second and third are the conditions of incentive compatibility and the first is the condition of individual rationality for the second player.

When there are only two states of nature S. Sorin (f) proved that an equilibrium for the un-discounted game exists. Sorin did this for a special kind of joint plan equilibrium known as an independent and twosafe joint plan equilibrium. Independent means that every $\gamma_{v} \in \Delta(I \times J)$ in the joint plan is generated independently by the product of a member $\sigma_{v}$ of $\Delta(I)$ with a member $\tau_{v}$ of $\Delta(J)$. Two-safe means that for every posterior probability $v \in V$ the second player's strategy $\tau_{v}$ guarantees to him his min-max payoff $b^{*}(v)$ against any action of the first player in the zero-sum game represented by the matrix $B(v)$.

In [17] the present authors extended Sorin's result to arbitrarily many states of nature. This had been accomplished through the use of (some of) the topological concepts and results discussed here in Section 2

\subsection{Second level of difficulty}

In the second level of difficulty one doesn't assume perfect monitoring by either player concerning what the other player has done, however one does assume that the perception of the second player is independent of the state of nature. The proof of equilibrium existence for this level is due to J. Renault ([14]). It, too, depended on the use of (modified) topological results from [17], and on the game-theoretic side involved essential new concepts described below.

The most important contribution of Renault to the second, third, and fourth levels of difficulty is his solution to the problem of statistical control. Like the first level of difficulty, the players find a cooperative agreement consisting of joint plans in which the first player signals to the second player which joint plan will be used. A joint plan equilibrium in this context is defined similarly to the first level of difficulty, except that the ability to perceive deviation must be worked into the definition. Because the players have only limited ability to communicate and perceive, there are significant problems to any such cooperative agreement. How does the uninformed player know which joint plan should be used? How does he know if the informed player is performing according to the plan?

Renault's solution involves an alternating sequences of playing and communicating phases. In the limit, the length of the communicating phases are insignificant compared to the playing phases, but the lengths of both phases goes toward infinity in the limit. In the communicating phases the informed player must convey to the uninformed player which joint plan will be performed. During the playing phases the uninformed player engages in random but increasingly rare "spot checks" to make sure that the informed player is performing according to the plan. In the playing phases they perform the actions corresponding to the joint plans (except for the occasional "spot check"). Required is punishment that lasts long enough to be effective, yet always terminates to allow for the possibility that it was an honest player who was punished. The probability that an honest player will be punished infinitely many times must be zero, while with probability one any attempt to gain a positive $\epsilon$ from deviation must be punished infinitely often. 
To make matters worse, there is always a residual possibility that the uninformed player will punish the informed player incorrectly simply because he misinterpreted the message in the communicating phase. While punishment is performed the second player must continue to listen to the first player during the communication phases. Incorporating all of these goals simultaneously was a significant achievement by Renault, which set up the possibility of solving the third and fourth level of difficulty.

\subsection{The third and fourth levels of difficulty}

The third level of difficulty involves no assumptions on the perception of the players other than the ability of the informed player to send two distinct messages to the uninformed player that are non-revealing with respect to the state of nature.

Now we present the signaling model of the third and fourth levels of difficulty. There are two sets of finite signals $R$ and $S$, received by the first and second players, respectively. There is a stochastic signaling function, $\Lambda: K \times I \times J \rightarrow \Delta(R \times S)$. After each stage in which $i \in I$ and $j \in J$ were played, and $k$ is the state of nature, a member of $R$ and a member of $S$ is determined by $\Lambda(k, i, j)$ and communicated to Player One and Player Two, respectively. The only knowledge the players have of the moves of their opponents is through their observations of the sets $R$ and $S$, and the only knowledge Player Two has of the state of nature is from the initial probability $p_{0}$ and the received sequence of signals in $S$. We will assume that both players can deduce their past behavior from the signals they receive.

For every $j \in J$ let $\Lambda^{j}:(K \times I) \rightarrow \Delta(S)$ be defined so that $\Lambda^{j}(k, i)$ is the marginal probability on the signals $S$ determined by the moves $i \in I, j \in J$, and the state $k \in K$. It represents the probability with which Player Two receives a signal $s \in S$ if Player Two chooses $j$, Player One chooses $i$, and the state of nature is $k$. A $\sigma \in \Delta(I)^{K}$ represents a choice, dependent on the state of nature, of a mixed strategy by Player One. For every $\sigma \in \Delta(I)^{K}$ with $\sigma=\left(\sigma^{k} \mid k \in K\right)$ let $\bar{\Lambda}^{j}: \Delta(I)^{K} \rightarrow \Delta(S)^{K}$ be the $|K|$-tuple of probabilities on $S$ determined by $\bar{\Lambda}^{j}(\sigma)^{k}:=\sum_{i \in I} \sigma_{i}^{k} \Lambda^{j}(k, i)$. For every $L \subset K$ define

$$
N R(L):=\left\{\sigma \in \Delta(I)^{K} \mid \forall j \forall k, k^{\prime} \in L \bar{\Lambda}^{j}(\sigma)^{k}=\bar{\Lambda}^{j}(\sigma)^{k^{\prime}}\right\} .
$$

$N R(L)$ are those state dependent choices that yield the same distribution on the signals $S$ no matter what Player Two does and no matter which state in $L$ was chosen. For every $p \in \Delta(K)$ define $\operatorname{supp}(p):=\{k \in$ $\left.K \mid p^{k}>0\right\} \subset K$.

For every $\sigma \in \Delta(I)^{K}$ and $\tau \in \Delta(J)$ define $\sigma A \tau \in \mathbb{R}^{K}$ (respectively $\sigma B \tau \in \mathbb{R}^{K}$ ) as $(\sigma A \tau)^{k}:=$ $\sigma^{k} A^{k} \tau$, so that for every $p \in \Delta(K)$ we have $p \cdot \sigma A \tau=\sum_{k \in K} p^{k}\left(\sigma^{k} A^{k} \tau\right) . \sigma A \tau$ is the payoff vector for Player One, a payoff for each state, resulting from her choice of $\sigma$ combined with Player Two's choice of $\tau$.

Define the function $a^{*}$ (not necessarily continuous) by

$$
a^{*}(p)=\max _{\sigma \in N R(\operatorname{supp}(p))} \min _{\tau \in \Delta(J)} p \cdot \sigma A \tau=\min _{\tau \in \Delta(J)} \max _{\sigma \in N R(\operatorname{supp}(p))} p \cdot \sigma A \tau
$$

where we set $a^{*}(p)=-\infty$ if $N R(\operatorname{supp}(p))=\emptyset$.

In the zero-sum context, these games were studied also by Aumann and Maschler [4]. Aumann and Maschler showed that the value of the game (in generality of the fourth level of difficulty) to the informed player is also cav $\left(a^{*}\right)\left(p_{0}\right)$, where $p_{0}$ is the initial probability on the states of nature. (See also [11] and [12].)

For a subset $L \subseteq K$ we will perceive $\Delta(L)$ both as an independent simplex and as a sub-simplex of $\Delta(K)$. For every $L \subset K$ with $N R(L) \neq \emptyset$, define the function $a_{L}^{*}$ on $\Delta(L)$ by

$$
a_{L}^{*}(p)=\max _{\sigma \in N R(L)} \min _{\tau \in \Delta(J)} p \cdot \sigma A \tau=\min _{\tau \in \Delta(J)} \max _{\sigma \in N R(L)} p \cdot \sigma A \tau .
$$

We have that $a_{L}^{*}$ is continuous and $a^{*}=\max _{L \subseteq K} a_{L}^{*}$.

The difficulty in proving equilibrium existence for a state dependent $\Lambda$ is the following. For every $L \subset K$ with $N R(L) \neq \emptyset, q \in \Delta(L)$ and $\tau \in \Delta(J)$, we know that there exists a $\sigma \in N R(L)$ such that $q \cdot \sigma A \tau \geq a_{L}^{*}(q)$. But individual rationality for the first player requires that the equilibrium payoff $y \in \mathbb{R}^{K}$ 
obey $y \cdot q \geq a^{*}(q)$ for all $q \in \Delta(K)$. This problem was not encountered by Renault ([14]) because state independence of the $\Lambda^{j}$ implied that $a^{*}=a_{K}^{*}$.

We define a family of special subsets of $K$ we call the family of non-extendible sets. A subset $L$ of $K$ is extendible to a larger subset $N$ if every member of $N R(L)$ can be extended to a member of $N R(N)$ yielding for every move $j \in J$ the same distribution in $\Delta(S)$. A subset $L \subset K$ is non-extendible if $N R(L) \neq \emptyset$ and there exits no strictly larger subset $N$ with $L$ extendible to $N$.

The usefulness of the family of non-extendible sets is two-fold. First, if nature chooses the state $k$ and $k$ is in a non-extendible set $L$ then there will be a way for the first player to demonstrate to the second player with certainty in the limit that the state of nature is indeed in $L$ without revealing any information concerning which member of $L$ it might be. Second, though the function $a^{*}$ is defined to be the maximum of the $a_{L}^{*}$ for all subsets $L \subset K$, we obtain $a^{*}$ also by restricting the maximum to only those $L$ that are nonextendible. An important property of the family of non-extendible sets is that it is closed under intersection. For any $p \in \Delta(K)$ define $F(p)$ to be the minimal non-extendible set containing $\operatorname{supp}(p)$, with $F(p)=K$ if there is no such set.

For every non-extendible set $L$ we work with a concept of joint plan equilibrium relative to $L$. Due to the signaling structure, our definition of such joint plan equilibria cannot be simple. First, because Player Two has limited ability to observe her behavior, Player One has much opportunity to cheat on any joint plan. We must require of the players' behavior that if Player One is asked to perform some $\sigma \in \Delta(I)^{K}$ then Player One is already choosing a strategy that maximizes her payoff inside of $L$ with respect to all the other strategies in $\Delta(I)^{K}$ that produce for every move $j$ of Player Two the same distribution on the signals $S$. (We assume that at random stages Player Two will perform all moves with equal probability, just to make sure that Player One is performing according to the plan.) On the other hand, Player One is also receiving signals in $R$ and does not know exactly what Player Two is doing. The easiest way to introduce this aspect of the game into the solution is require of Player Two's strategies that they maximize his payoff in response to what Player One is doing. This property, used by Renault, is called two-best-reply and is a parallel to the two-safe property of [19]. Such combinations of strategies, called proto-joint-plans, describe only the behavior on subsets of the playing stages. The proto-joint-plans must be combined into a way for the players to behave on all the playing stages if some probability $p \in \Delta(L)$ is given. We call these combinations non-revealing joint plans. Then for every initial probability $p_{0} \in \Delta(L)$ a lottery will be performed by Player One that determines one of possibly many posterior probabilities, for every such posterior $p$ is associated a non-revealing joint plan that determines how the players should act in the future in the stages that will matter for their average payoffs. Finally these non-revealing joint plans for various $p$ in a set $V \subseteq \Delta(L)$ such that the initial $p_{0}$ is a convex combination of the $V$ will define a joint plan for $p_{0}$. Incentive compatibility and individual rationality are required from the joint plan to make it a joint plan equilibrium, just as with the first level of difficulty.

The main problem lies with a comparison of the joint plan equilibria coming from different nonextendible $L$. Domination of the function $a_{L}^{*}$ is the natural individual rationality condition for each $L$, and for which we can prove existence of joint plan equilibria using the old methods. But we need our payoff vectors to dominate the function $a^{*}$. How can we put together joint plan equilibria from the different non-extendible $L$ to create a joint plan equilibrium for the original game? The putting together of the solutions from the different members of $\mathcal{L}$ is accomplished by Theorem 1 But notice that Theorem 1 assumes that the inverse images of the appropriate correspondence are convex. This convexity assumption is implied by the ability of the first player to send distinct signals that reveal nothing about the state, the property that differentiates the third level from the fourth level of difficulty.

\section{Principal-Agent Contracts}

Our topological results can be applied to economic situations broader than that of repeated games of incomplete information, in particular to principal-agent situations. A typical principal-agent situation is that of the relationship between the owner of a firm (the principal) and its manager (the agent). The owner employs 
the manager, both are interested in the success of the firm, but their interests do not coincide and the agent has information on the firm that the owner does not have.

A mathematical abstraction of principal-agent situations follows. There are two persons involved, a principal and an agent. There is a finite set $K$ of possibilities called states (of nature), a convex and compact set $X$ of joint actions, and a set of signals. Nature chooses a state in $K$ according to an initial probability distribution $p_{0}$ in the interior of the simplex $\Delta(K)$ (meaning positive probability given to each state). Soon after nature chooses some $k \in K$ the agent will learn of nature's choice in $K$ but the principal is not informed beyond the initial probability distribution $p_{0}$. After knowing the state of nature the agent sends a signal to the principal. A contract is an assignment of a joint action to each signal sent by the agent along with a state dependent stochastic rule for how that signal should be chosen. The payoffs to both players are determined by the choice of joint action and by the state of nature. The principal can obtain additional information concerning the state of nature (beyond the initial distribution $p_{0}$ ) only through the signal sent from the agent. The agent and principal are not bounded by their contract to play the joint action until after the principal has received the signal from the agent. Given some reasonable assumptions on the structure of the payoffs we will show that there exists a contract in equilibrium.

We make the assumption that there is a family $\mathcal{L}$ of subsets of the states $K$ such that $\bigcup \mathcal{L}=K$ and, moreover, if the chosen state does lie in a member $L$ of the family $\mathcal{L}$ then the agent has the ability to prove to the principal that the chosen state of nature does lie in this subset without necessarily revealing any additional information about the state of nature. This assumption only strengthens our result, as one could always assume that $\mathcal{L}$ is the singleton $\{K\}$. Any signal sent by the agent will have attached to it a subset $L \in \mathcal{L}$ such that this signal can be sent from any state in this subset but from no state outside of this subset.

Caratheodory's Theorem states that if a vector $r$ in $n$ dimensional Euclidean space is in the convex hull of a finite set then it is also in the convex hull of a subset of cardinality no more than $n+1$. Due to this theorem, we assume without loss of generality that for every $L \in \mathcal{L}$ there is a finite subset $S_{L}$ of signals with $\left|S_{L}\right|=|L|$. Let $S$ be the disjoint union $\bigcup_{L \in \mathcal{L}} S_{L}$.

Every choice of a joint action $x \in X$ corresponds to a set of payoffs for the agent. The same may be true for the principal, but such a quantity will not be essential to defining equilibrium behavior. For the agent we associate with every $x \in X$ a vector payoff $r_{x} \in \mathbb{R}^{K}$ such that the agent will receive the quantity $r_{x}^{k}$ if action $x$ is taken and $k$ is the state of nature. We assume also that the vectors $r_{x}$ respect the convex structure of the set $X$, namely that if $x=\lambda x_{1}+(1-\lambda) x_{2}$ then $r_{x}=\lambda r_{x_{1}}+(1-\lambda) r_{x_{2}}$.

A contract is an associated joint action $x_{s} \in X$ for each $s \in S_{L}$ and a collection $w=\left(w_{k} \in \Delta(S) \mid k \in\right.$ $K)$ of state dependent distributions on the signals such that if $w_{k}(s)>0$ and $s \in S_{L}$ then $k \in L$. The vector $y \in \mathbb{R}^{K}$ defined by $y^{k}=\sum_{s \in S} w_{k}(s) r_{x_{s}}^{k}$ is called the expectation vector of the contract.

Given a contract define a signal $s$ to be used if $w^{k}(s)>0$ for some $k \in K$. For every contract and signal $s \in S_{L}$ that is used there is a probability distribution $p_{s}$ on $\Delta(L)$ defined by $p_{s}(k):=\frac{w_{k}(s)}{\sum_{l \in L} w_{l}(s)}$. This is the conditional probability distribution on $L$ defined by Bayes Rule.

Acceptability conditions For any set $L \in \mathcal{L}$ there is a correspondence $F_{L}: \Delta(L) \rightarrow X$. The interpretation is that if the agent sends the signal $s \in S_{L}$ and $p_{s}$ is the conditional probability on $L$ induced by the contract then $F_{L}(p)$ are the joint actions that are acceptable to the principal.

For any $L \in \mathcal{L}$ and $q \in \Delta(L)$ we assume that there is a value $f_{L}(q)$ such that if the agent were informed by nature only of the $L$ and $q$ then the agent could demand the value of $f_{L}(q)$ for herself without the need of any contract with the principal.

Because of the asymmetry of the information received by the principal and the agent, the mathematical conditions defining what is acceptable to the agent must be very different from those defining what is acceptable to the principal, (which is why we didn't need to quantify what is acceptable to the principal). After a signal has been sent by the agent there is only one distribution on the states of nature that matters to the principal, namely that calculated by Bayes rule. On the other hand, because the agent knows precisely the state of nature all probability distributions in $\Delta(K)$ are relevant (not only the $K$ extremal points of $\Delta(K)$ giving all weight to a single state). Given any initial probability distribution $p_{0}$ in the interior of 
$\Delta(K)$ the agent can choose to receive its knowledge of the state in two steps, first through membership in any set $V$ such that $p_{0}$ is in the convex hull of $V$ with $p_{0}=\sum_{v \in V} \lambda_{v} v$ for all $\lambda_{v}$ positive and $\sum_{v \in V} \lambda_{v}=1$ and then followed by the agent's learning of the precise member of $K$. For any $q \in \Delta(K)$ there will be such a set $V$ that includes $q$. Therefore any contract that is acceptable to the agent must have an expectation vector $y$ that satisfies $y \cdot q^{L} \geq f_{L}(q)$ for all pairs $L \in \mathcal{L}$ and $q \in \Delta(L)$.

Equilibrium: There are three ways that a contract can fail to be in equilibrium. First, after the signal is sent by the agent the joint action suggested may be unacceptable to the principal. Second, the expectation vector of the contract may be unacceptable to the agent. Third, at some chosen state $k$ the agent may prefer the payoff associated with one signal over that associated with some other signal sent with positive probability.

Definition 5 Given an initial probability distribution $p_{0}$ in the interior of $\Delta(K)$ a contract is an equilibrium if and only if

(1) $x_{s} \in F_{L}\left(p_{s}\right)$ for all used $s \in S_{L}$

(2) the expectation vector $y \in \mathbb{R}^{K}$ satisfies $y^{L} \cdot q \geq f_{L}(q)$ for every $L \in \mathcal{L}$ and $q \in \Delta(L)$,

(3a) $r_{x_{s}}^{k}=y^{k}$ for all used $s \in S$ and $k \in K$ with $p_{s}^{k}>0$, and

(3b) $r_{x_{s}}^{k} \leq y^{k}$ for all used $s \in S$ and $k \in K$.

Theorem 4 If every correspondence $F_{L}$ is u.s.c., non-empty and convex valued, every function $f_{L}$ is lower-semicontinuous, and for every $L \in \mathcal{L}$ and $p, q \in \Delta(L)$ there is an $x \in F_{L}(p)$ such that $r_{x} \cdot q \geq f_{L}(q)$, then for every initial probability $p_{0}$ in the interior of $\Delta(K)$ there is a contract in equilibrium.

PRoof. For every $L \in \mathcal{L}$ the correspondence $c \tilde{F}_{L}$ (as defined in Theorem 2) represents the contracts in equilibrium with respect to the set $L$, (meaning that only members of $S_{L}$ and the correspondence $F_{L}$ are used, $y^{L} \cdot q \geq f_{L}(q)$ is required for all $q \in \Delta(L)$, and the equalities and inequalities of (3a) and (3b) apply only to the states $k \in L)$. By Theorem 2 for every $L \in \mathcal{L}$ the correspondence $c \tilde{F}_{L}$ has the spanning property for the set $\Delta(L)$. With $U_{L}$ defined to be $\left\{y \mid y^{L} \cdot q \geq f_{L}(q) \forall q \in \Delta(L)\right\}$ and $b \in \mathbb{R}$ a quantity larger than any payoff defined in the game, Theorem 1 implies the existence of an equilibrium (with $c \tilde{F}_{L}$ replacing the $F_{L}$ of the theorem).

In connection with sections 2.6 and 3.5 we would like to mention that a further advance along the lines discussed in Section 3.5 may depend on proving and being able to use an appropriate parametric version of Borsuk-Ulam theorem. A work in progress in this direction is undertaken by Thomas Schick and the present authors. A part of it is contained in the preprint "A parametrized version of Borsuk-Ulam theorem" (available on arXiv).

\section{References}

[1] Alexandroff, P., (1932). Dimensionstheorie. Ein Beitrag zur Geometrie ger abgeschlossenen Mengen, Math. Ann., 106, 161-238.

[2] Aumann, R. and Maschler, M., (1966). Game Theorethic Aspects of Gradual Disarmament, Mathematica, V. 1V. 55, Princeton, N. J.

[3] Aumann, R. and Maschler, M., (1967). Repeated games of incomplete information: A survey of recent results, Princeton: Mathematica ST-143,

[4] Aumann, R. and Maschler, M., (1995). Repeated Games with Incomplete Information, with the collaboration of R. Stearns, M. I. T. Press, Cambridge, MA. 
[5] Aumann, R. and Maschler, M. and Stearns, R., (1968). Repeated games of incomplete information: An approach to the non-zero-sum case, Princeton: Mathematica ST-143,

[6] Blackwell, D., (1956). An Analog of the Minimax Theorem for Vector Payoffs, Pacific J. of Mathematics, 6, 1-8.

[7] Eilenberg, S. and Steenrod, N., (1952). Foundations of Algebraic Topology, Princeton Univ. Press, Princeton, N.J.

[8] Harsanyi, J., (1967/68). Games with incomplete information played by Bayesian players, parts I-III, Management Science, 14, 159-182, 320-334, 486-502.

[9] Izydorek, M. and Jaworowski, J., (1995). Antipodal coincidence for maps of spheres into complexes, Proc. Amer. Math. Soc., 123, 6, 1947-1950.

[10] Joshi, K. D., (1973). A non-symmetric generalization of the Borsuk-Ulam theorem, Fundamenta Mathematicae, 80, 13-33.

[11] Kohlberg, E., (1975). Optimal Strategies in Repeated Games with Incomplete Information, International Journal of Game Theory, 4, 7-24.

[12] Mertens, J.-F., Sorin, S., and Zamir, S., (1994). Repeated Games, Core Discussion Papers, 9420-9422, Université Catholique de Louvain.

[13] Olędzki, J., (1978). On a generalization of the Borsuk-Ulam theorem, Bulletin de l'Académie Polonaise Sciences, 26, 157-162.

[14] Renault, J., (2000). On Two-player Repeated Games with Lack of Information on One Side and State-independent Signalling, Mathematics of Operations Research, 25, 4, 552-572.

[15] Ščepin, E. V., (1974). A certain problem of L. A. Tumarkin (Russian). Dokl. Akad. Nauk SSSR, 217, 42-43.

[16] Sieklucki, K., (1969). A generalization of the Borsuk-Ulam theorem on antipodal points, Bulletin de l'Académie Polonaise Sciences, 17, 629-631.

[17] Simon, R., Spież, S. and Toruńczyk, H., (1995). The Existence of Equilibria in Certain Games, Separation for Families of Convex Functions and a Theorem of Borsuk-Ulam Type, Israel Journal of Mathematics, 92, 1-21.

[18] Simon, R., Spież, S. and Toruńczyk, H., (2002). Equilibrium existence and topology in some repeated games with incomplete information, Transactions of the American Mathematical Society, 354, 5005-5026

[19] Sorin, S., (1983). Some Results on the Existence of Nash Equilibria for Non-zero-sum Games with Incomplete Information, International Journal of Game Theory, 12, 193-205.

[20] Spanier, E. H., (1966). Algebraic Topology, McGraw-Hill, New York.

[21] Тихомиров, В. М.,(1975). Некоторые вопросы теории приближении, (Russian). Moscow Univ. Press,

\section{Robert S. Simon}

Department of Mathematics, London School of Economics,

Houghton St.,

WC2A 2AE London, U.K.

R.S.Simonelse.ac.uk

\section{Stanisław Spież}

Institute of Mathematics, Polish Academy of Sciences, Śniadeckich 8, P.O.B. 137, 00-950 Warszawa, Poland S.Spiez@impan.gov.pl

\section{Henryk Toruńczyk}

Institute of Mathematics, Warsaw University,

Banacha 2, 02-097 Warszawa, Poland

torunczy@mimuw.edu.pl 